\title{
Synbiotic Supplementation Improves Obesity Index and Metabolic Biomarkers in Thai Obese Adults: A Randomized Clinical Trial
}

\author{
Chaiyavat Chaiyasut $^{1}(\mathbb{D})$, Bhagavathi Sundaram Sivamaruthi ${ }^{1, *(D)}$, Periyanaina Kesika ${ }^{1}\left(\mathbb{D}\right.$, Suchanat Khongtan ${ }^{1}$, \\ Nanticha Khampithum ${ }^{1}$, Subramanian Thangaleela ${ }^{1}$, Sartjin Peerajan ${ }^{2}$, Akkarach Bumrungpert ${ }^{3,4}$ (D), \\ Khontaros Chaiyasut ${ }^{5}$, Sasithorn Sirilun ${ }^{1}$ and Phakkharawat Sittiprapaporn ${ }^{6, *}$ D
}

Citation: Chaiyasut, C.; Sivamaruthi, B.S.; Kesika, P.; Khongtan, S.; Khampithum, N.; Thangaleela, S.; Peerajan, S.; Bumrungpert, A.; Chaiyasut, K.; Sirilun, S.; et al. Synbiotic Supplementation Improves Obesity Index and Metabolic Biomarkers in Thai Obese Adults: A Randomized Clinical Trial. Foods 2021, 10, 1580. https://doi.org/ $10.3390 /$ foods 10071580

Academic Editor: Paula C. Castilho

Received: 22 May 2021

Accepted: 5 July 2021

Published: 7 July 2021

Publisher's Note: MDPI stays neutral with regard to jurisdictional claims in published maps and institutional affiliations.

Copyright: (c) 2021 by the authors. Licensee MDPI, Basel, Switzerland. This article is an open access article distributed under the terms and conditions of the Creative Commons Attribution (CC BY) license (https:// creativecommons.org/licenses/by/ $4.0 /)$
1 Innovation Center for Holistic Health, Nutraceuticals, and Cosmeceuticals, Faculty of Pharmacy, Chiang Mai University, Chiang Mai 50200, Thailand; chaiyavat@gmail.com (C.C.); p.kesika@gmail.com (P.K.); suchanat_k@cmu.ac.th (S.K.); nanticha_khampithum@cmu.ac.th (N.K.); leelasubramanian@gmail.com (S.T.); sasithorn.s@cmu.ac.th (S.S.)

2 Health Innovation Institute, Chiangmai 50200, Thailand; s.peerajan@gmail.com

3 Mahidol Nutrition Society, Faculty of Public Health, Mahidol University, Bangkok 10400, Thailand; abnutrition@yahoo.com

4 Research Center of Nutraceuticals and Natural Products for Health \& Anti-Aging, College of Integrative Medicine, Dhurakij Pundit University, Bangkok 10210, Thailand

5 Institute of Research and Development, Chiang Mai Rajabhat University, Chiangmai 50300, Thailand; khontaros_cha@cmru.ac.th

6 Neuropsychological Research Laboratory, Department of Anti-Aging and Regenerative Science, School of Anti-Aging and Regenerative Medicine, Mae Fah Luang University, Bangkok 11120, Thailand

* Correspondence: sivamaruthi.b@cmu.ac.th (B.S.S.); wichian.sit@mfu.ac.th (P.S.); Tel.: +66-53-944-340 (B.S.S.)

Abstract: The cluster of metabolic disorders includes obesity, dyslipidemia, hypertension, and glucose intolerance, increasing the risk of developing cardiovascular diseases and type 2 diabetes. Evolving proofs suggest an essential role of microbiota in human health and disease, including digestion, energy and glucose metabolism, immunomodulation, and brain function. The frequency of overweight is increasing, and the main causes for this are highly processed foods and less active lifestyles. Research is underway to unravel the probable relationship between obesity and intestinal microbiota. Here, we propose a method to understand and elucidate the synergistic function of prebiotics and probiotics in treating obesity. The biomarkers of obesity, such as cholesterol, gut permeability, oxidative stress, bacterial toxins, cytokines, and short-chain fatty acids, were analyzed in Thai obese individuals after being supplemented with a synbiotic preparation containing Lactobacillus paracasei, Bifidobacterium longum, Bifidobacterium breve, inulin, and fructooligosaccharide. The results reveal that the supplementation of synbiotics significantly altered the obesity-associated biomarkers in an appositive way. Further studies are warranted to use synbiotics as an adjuvant therapy for the management of obesity-related health issues.

Keywords: obesity; synbiotics; Lactobacillus; Bifidobacterium; inulin; fructooligosaccharide

\section{Introduction}

Obesity is one of the major health issues worldwide, leading to other health issues such as cardiovascular diseases, diabetes, and hypertension' which result in morbid obesity. A long-term imbalance in energy consumption, an irregular diet, altered gut microbiota, environmental factors, and genetic makeup are the primary causes of obesity [1]. According to a WHO report, about 650 million adults are obese, and 1.9 billion are overweight. Of these, possibly 38 million children (less than five years old) are obese [2].

The intestinal microbiota composition has a critical role in obesity [1]. For example, the Firmicutes to Bacteroidetes proportion was found to be higher in overweight/obese people compared to ordinary people. Energy absorption and storage may be associated with the 
balance of Firmicutes in intestinal microbiota [3,4]. The dysbiosis in intestinal microbiota is associated with cell homeostasis changes and affects the integrity of tight junctions, resulting in a decline in gut permeability [5]. Dysbiosis also influences inflammation, insulin resistance, and fat deposition, leading to the development of obesity. It increases the bacterial toxic load (i.e., lipopolysaccharide) in the host [6].

Probiotic bacteria are live microorganisms that confer a health benefit on the host when administered in suitable amounts. Recent studies have highlighted the beneficial effects of probiotics supplementation in hosts with metabolic disorders, cognitive declines, and cancers via the positive regulation of gut microbiota [1,7-9]. The supplementation of synbiotics (a mixture of probiotics and prebiotics) may effectively improve intestinal microbiota composition compared to probiotics or prebiotics supplements [10].

The combination of Lactobacillus and Bifidobacterium, along with prebiotics, could provide synergic effects to the host. So far, studies on the influence of the supplementation of synbiotic preparations containing Lactobacillus paracasei, Bifidobacterium longum, Bifidobacterium breve, inulin, and fructooligosaccharide on cholesterol profiles, cytokines, markers of leaky gut, antioxidant levels, and short-chain fatty acids (SCFAs) contents in Thai obese adults have not yet been reported. Thus, we aimed to study the effect of a synbiotic intervention on the biomarkers of cholesterol, gut permeability, oxidative stress, bacterial toxins, cytokines, and SCFAs in Thai obese subjects.

\section{Materials and Methods}

\subsection{Study Design and Subjects}

The study on the effects of synbiotics on obesity was conducted with randomized, double-blind placebo-controlled trials of Thai obese adults. The participants of this study provided their informed consent for participation before they joined the study. The Good Clinical Practices were followed in the study. The Ethics Committee of Mae Fah Luang University approved the study protocol (Code: REH-62151).

The inclusion criteria included Thai obese adults (BMI $\geq 25 \mathrm{~kg} / \mathrm{m}^{2}$ ) according to the Asia-Pacific criteria, aged 18-65 years, who were willing to participate and complete the study. Subjects with kidney diseases, cardiovascular issues, gouty arthritis, and gastrointestinal tract discomforts were excluded from the study.

Randomization was conducted with computer-generated codes using Random Allocation Software version 1.0.0 (Isfahan, Iran) [11]. The researchers and participants were blinded to the group assignment. Participants were randomized to receive either a synbiotic preparation (Lactobacillus paracasei, Bifidobacterium longum, Bifidobacterium breve, inulin, and fructooligosaccharide) or placebo for 12 week-long supplementations. After 12 weeks of supplementation, participants were asked to return for follow-up visits. The study flowchart and enrollment are described in Figure 1.

\subsection{Treatment}

Aluminum foil sachets containing $5 \times 10^{10} \mathrm{CFU}$ of probiotics $\left(2 \times 10^{10} \mathrm{CFU}\right.$ of Lactobacillus paracasei, $1 \times 10^{10} \mathrm{CFU}$ of Bifidobacterium longum, $2 \times 10^{10} \mathrm{CFU}$ of Bifidobacterium breve) and prebiotics (5 $\mathrm{g}$ of inulin and $5 \mathrm{~g}$ of fructooligosaccharide) were provided to the subjects in the synbiotic group. The concentration of Bifidobacterium breve was decided based on the anti-obesity effects of $B$. breve reported in a randomized, double-blind, placebo-controlled trial [12]. The combination of synbiotic and the concentration of other probiotics used in this study were based on our results (unpublished data). The probiotics were received from Lactomason Co., Ltd., (Gyeongsangnam-do, South Korea), and prebiotics were purchased from BENEO-Orafti S.A., (Oreye, Belgium). Those in the placebo group were provided with $10 \mathrm{~g}$ of corn starch. All subjects were instructed to regularly take the supplementation by dissolving the contents of one sachet in a glass of water before breakfast. 


\section{Enrollment}

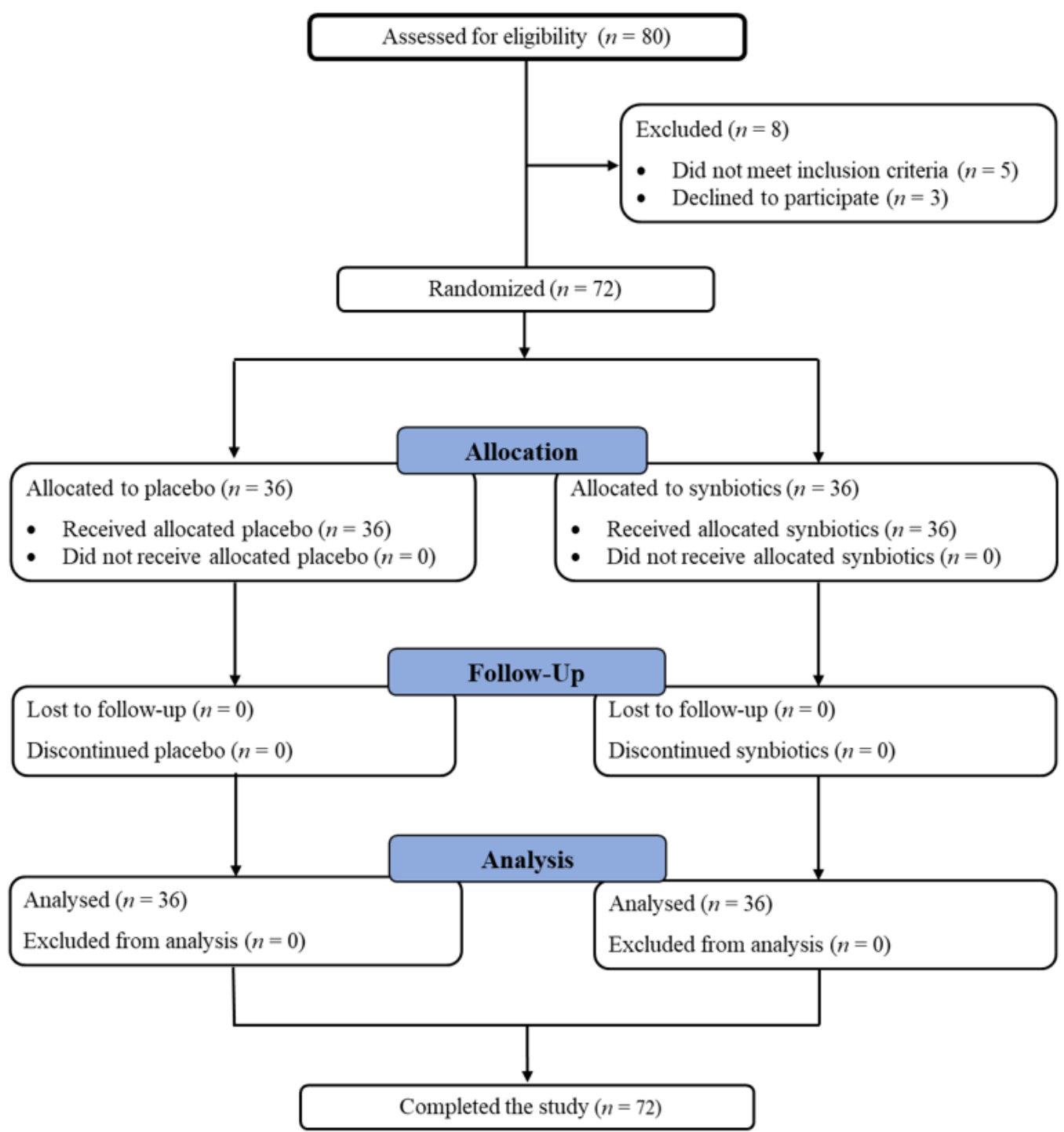

Figure 1. The study flowchart and enrollment.

\subsection{Assessments}

\subsubsection{Clinical Data}

The subjects' personal history was assessed, including education, physical activities, smoking and alcohol drinking habits, and pharmacological treatments.

Demographic characteristics, including age, diabetes, alcohol drinking, and obesity index, were recorded manually. Body weight, body mass index (BMI), body fat, visceral fat, basal metabolic rate (BMR), and muscle were measured using an electronic scale (Picooc ${ }^{\circledR}$, Model S1 Pro, Beijing, China).

\subsubsection{Laboratory Data}

Blood, fecal, and urine samples were collected at baseline and the end of the study (Figure 2). The biochemical analyses including total cholesterol (TC), HDL-cholesterol (HDL-C), LDL-cholesterol (LDL-C), triglycerides (TG), and fasting blood sugar (FBS) levels were determined from blood using the automated machine at AMS Clinical Service Center, Chiang Mai University, Chiang Mai, Thailand. Other biomarkers in the blood such as high sensitivity C-reactive protein (hs-CRP), immunoglobulin A (IgA), lipopolysaccharides (LPS), zonulin (ZO-1), and inflammatory chemokines/cytokines were determined 
using an ELISA commercial kit (OriGene Technologies, Rockville, MD, USA for hs-CRP, Elabscience $^{\circledR}$, Houston, TX, USA for IgA, MyBioSource ${ }^{\circledR}$, San Diego, CA, USA for LPS and IDK $^{\circledR}$, Bensheim, Germany for ZO-1). Plasma total antioxidant capacity (TAC) was determined by a 2,2'-Azinobis (3-ethylbenzothiazoline-6-sulfonic acid) (ABTS) radical scavenging capacity assay $[13,14]$. The determination of malondialdehyde (MDA) was performed with the thiobarbituric acid reactive substances (TBARS) method $[15,16]$. The dismutation of superoxide radicals was determined using the assay of superoxide dismutase (SOD) [17], and reduced glutathione (GSH) in the plasma was determined using the recycling assay of 5,5'-dithiobis (2-nitrobenzoic acid) (DTNB) [18].

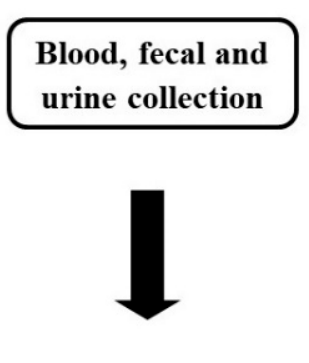

$\mathbf{0}$
Blood, fecal and urine collection

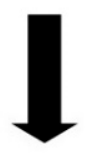

12

Week

Baseline

End Synbiotic supplementation

Figure 2. The timeline of this study.

Fecal samples were collected to determine the short-chain fatty acids using highperformance liquid chromatography (HPLC) according to the following conditions: Shodex SH1011 as a column, $5 \mathrm{mM}$ sulfuric acid as the mobile phase, with a flow rate of $0.6 \mathrm{~mL} / \mathrm{min}$ at $210 \mathrm{~nm}$ and $75^{\circ} \mathrm{C}[19,20]$, and putrefaction using HPLC with the following conditions: C18 $(4.6 \mathrm{~mm} \times 15 \mathrm{~cm})$ as a column, methanol: water $(60: 40 \mathrm{v} / \mathrm{v})$ as mobile phase, with a flow rate of $0.5 \mathrm{~mL} / \mathrm{min}$ at $200 \mathrm{~nm}$ [21-23].

Urine samples were used to determine intestinal permeability. The subjects were given mannitol and lactulose at a ratio of 1:2, dissolved in water. After taking mannitol and lactulose, subjects were asked to collect urine within $6 \mathrm{~h} \mathrm{[24].} \mathrm{We} \mathrm{measured} \mathrm{the} \mathrm{total} \mathrm{urine}$ volume from each subject and analyzed the intestinal permeability using a colorimetric commercial kit (EnzyChrom ${ }^{\mathrm{TM}}$, BioAssay, Hayward, CA, USA). Neuroinflammation markers in the urine, such as quinolinic acid (QA) and 5-hydroxyindoleacetic acid (5-HIAA), were determined using an ELISA commercial kit (Fivephoton Biochemicals ${ }^{\mathrm{TM}}$, San Diego, CA, USA for QA and Immusmol, Bordeaux, France for 5-HIAA).

\subsubsection{Statistical Analyses}

Demographics were continuously analyzed using a t-test and discrete data using exact values. Data were analyzed using the paired t-test of means using STATA version 15.1 (StataCorp, College Station, TX, USA) for Windows licensed to the Faculty of Pharmacy, Chiang Mai University, Chiang Mai, Thailand. A descriptive analysis of the collected parameters was expressed as an absolute number and percentage. The continuous variables were represented as mean \pm standard deviation (SD) or standard error of the mean (SEM) depending on their statistical distribution. The group's data were calculated using a t-test and Gaussian regression analysis. The minimum level of statistical significance was set to $p<0.05$ (two-tailed). 


\section{Results}

A total of 72 subjects completed the study. There were no differences between synbiotic and placebo groups in terms of the initial measurements of age, body weight, BMI, body fat, visceral fat, muscle, arm, waist, and hip circumferences, waist/hip ratio, blood urea nitrogen content, creatinine, aspartate aminotransferase, and alanine aminotransferase, except in their BMR (Table 1).

Table 1. Basic characteristics of the study subjects.

\begin{tabular}{cccc}
\hline Parameters & $\begin{array}{c}\text { Synbiotic Group } \\
(\mathbf{N}=\mathbf{3 6})\end{array}$ & $\begin{array}{c}\text { Placebo Group } \\
\mathbf{( N = 3 6 )}\end{array}$ & $p$-Value \\
\hline Age (years) & $54.78 \pm 1.92$ & $58.94 \pm 1.32$ & 0.078 \\
Body weight, cm & $69.09 \pm 1.90$ & $68.17 \pm 1.63$ & 0.712 \\
Body mass index, $\mathrm{kg} / \mathrm{m}^{2}$ & $28.97 \pm 0.77$ & $30.01 \pm 0.47$ & 0.248 \\
Body fat, \% & $33.09 \pm 1.18$ & $35.36 \pm 0.87$ & 0.125 \\
Visceral fat, \% & $14.18 \pm 0.88$ & $15.36 \pm 0.43$ & 0.223 \\
Muscle, \% & $56.48 \pm 3.85$ & $59.19 \pm 1.44$ & 0.497 \\
BMR (kcal) & $1409.42 \pm 31.93$ & $1323.04 \pm 23.86$ & 0.033 * \\
Arm circumference, cm & $30.71 \pm 0.49$ & $30.62 \pm 0.48$ & 0.893 \\
Waist circumference, cm & $94.73 \pm 1.92$ & $95.79 \pm 1.34$ & 0.651 \\
Hip circumference, cm & $103.09 \pm 1.38$ & $104.33 \pm 1.10$ & 0.486 \\
Waist/hip ratio & $0.92 \pm 0.01$ & $0.92 \pm 0.01$ & 0.94 \\
Diabetes, $\mathrm{n}(\%)$ & $7(19.44 \%)$ & $13(36.11 \%)$ & 0.188 \\
Alcohol drinking, $\mathrm{n}(\%)$ & $6(16.67 \%)$ & $4(11.11 \%)$ & 0.735 \\
Blood urea nitrogen $(\mathrm{mg} / \mathrm{dL})$ & $14.89 \pm 0.93$ & $16.86 \pm 1.84$ & 0.699 \\
Creatinine $(\mathrm{mg} / \mathrm{dL})$ & $1.09 \pm 0.09$ & $1.08 \pm 0.10$ & 0.964 \\
Aspartate aminotransferase (IU/L) & $25.43 \pm 4.62$ & $24.73 \pm 2.32$ & 0.744 \\
Alanine aminotransferase (IU/L) & $27.59 \pm 6.00$ & $23.68 \pm 2.97$ & 0.925 \\
\hline
\end{tabular}

* = Significant difference in $p$-value at 95\% confidence interval. The proportion was analyzed using an exact probability test, and the continuous demographic data were analyzed using a $t$-test. BMR: basal metabolic rate.

There were no changes in all studied parameters after 12 weeks in the placebo group compared with baseline values. In the synbiotic group, significant differences were observed after 12 weeks of supplementation in body weight, BMI, body fat, waist circumference, waist/hip ratio, HDL-C, LDL-C, IL-6, IL-10, IL-1 $\beta$, TNF- $\alpha$, IgA, LPS, and ZO-1 values compared to the baseline values. No significant changes were observed in visceral fat, muscle, BMR, arm and hip circumferences, TC, TG, and hsCRP values in the synbiotic group (Table 2).

The antioxidant systems (TAC, MDA, GSH, total SOD, and $\mathrm{Cu}, \mathrm{Zn}-\mathrm{SOD}$ ) of the subjects were documented. There were no statistically significant changes in the synbiotic and placebo groups after 12 weeks of supplementation (Table 3). The levels of butyric acid, propionic acid, acetic acid, and lactic acid were significantly changed after 12 weeks of synbiotic supplementation, whereas no changes were observed in the placebo group. The levels of lactulose, QA, the QA/5-HIAA ratio, cresol, and indole were significantly changed in the synbiotic group, which was not observed in the placebo group after 12 weeks (Table 3).

The significant changes in the studied parameters between the synbiotic and placebo groups after 12 weeks were calculated. The body weight, FBS, and cytokines, IgA, hsCRP, LPS, and QA levels were significantly altered compared to the placebo group (Tables 4 and 5). There were no notable changes in the rest of the studied parameters between the synbiotic and placebo groups. 
Table 2. Changes in the studied parameters within groups at different times, expressed as mean \pm SE.

\begin{tabular}{|c|c|c|c|c|c|c|}
\hline \multirow{2}{*}{ Parameters } & \multicolumn{2}{|c|}{ Synbiotic $(\mathrm{N}=36)$} & \multirow{2}{*}{$p$-Value } & \multicolumn{2}{|c|}{ Placebo $(\mathrm{N}=36)$} & \multirow{2}{*}{$p$-Value } \\
\hline & Baseline & 12 Weeks & & Baseline & 12 Weeks & \\
\hline Body weight, cm & $69.09 \pm 1.90$ & $67.45 \pm 1.85$ & $<0.001 *$ & $68.17 \pm 1.63$ & $67.71 \pm 1.71$ & 0.067 \\
\hline Body mass index, $\mathrm{kg} / \mathrm{m}^{2}$ & $28.97 \pm 0.77$ & $28.58 \pm 0.75$ & $0.017 *$ & $30.01 \pm 0.47$ & $30.13 \pm 0.58$ & 0.662 \\
\hline Body fat, $\%$ & $33.09 \pm 1.18$ & $31.96 \pm 1.20$ & $0.043 *$ & $35.36 \pm 0.87$ & $36.27 \pm 1.22$ & 0.310 \\
\hline Visceral fat, $\%$ & $14.18 \pm 0.88$ & $13.85 \pm 0.79$ & 0.162 & $15.36 \pm 0.43$ & $15.56 \pm 0.52$ & 0.445 \\
\hline Muscle, \% & $56.48 \pm 3.85$ & $56.08 \pm 3.81$ & 0.284 & $59.19 \pm 1.44$ & $59.01 \pm 1.42$ & 0.860 \\
\hline BMR (kcal) & $1409.42 \pm 31.93$ & $1411.27 \pm 29.61$ & 0.898 & $1323.04 \pm 23.86$ & $1309.68 \pm 24.87$ & 0.102 \\
\hline Arm circumference, $\mathrm{cm}$ & $30.71 \pm 0.49$ & $30.59 \pm 0.57$ & 0.808 & $30.62 \pm 0.48$ & $30.51 \pm 0.53$ & 0.795 \\
\hline Waist circumference, $\mathrm{cm}$ & $94.73 \pm 1.92$ & $92.76 \pm 1.84$ & 0.009 * & $95.79 \pm 1.34$ & $95.34 \pm 1.45$ & 0.648 \\
\hline Hip circumference, $\mathrm{cm}$ & $103.09 \pm 1.38$ & $102.50 \pm 1.30$ & 0.419 & $104.33 \pm 1.10$ & $103.84 \pm 1.31$ & 0.705 \\
\hline Waist/hip ratio & $0.92 \pm 0.01$ & $0.90 \pm 0.01$ & $0.018 *$ & $0.92 \pm 0.01$ & $0.92 \pm 0.01$ & 0.961 \\
\hline Total cholesterol (mg/dL) & $200.97 \pm 8.40$ & $195.50 \pm 6.48$ & 0.171 & $203.30 \pm 8.11$ & $199.97 \pm 7.67$ & 0.626 \\
\hline Triglyceride (mg/dL) & $150.24 \pm 16.04$ & $145.97 \pm 14.66$ & 0.469 & $148.64 \pm 11.04$ & $149.88 \pm 11.20$ & 0.893 \\
\hline HDL-cholesterol (mg/dL) & $50.21 \pm 2.42$ & $53.10 \pm 2.53$ & 0.030 * & $50.42 \pm 1.47$ & $50.91 \pm 2.56$ & 0.813 \\
\hline LDL-cholesterol (mg/dL) & $123.93 \pm 8.61$ & $112.66 \pm 6.62$ & 0.017 * & $123.35 \pm 7.35$ & $116.48 \pm 7.06$ & 0.295 \\
\hline FBS $(\mathrm{mg} / \mathrm{dL})$ & $111.79 \pm 7.44$ & $109.00 \pm 6.02$ & 0.373 & $109.68 \pm 6.76$ & $118.18 \pm 6.89$ & 0.084 \\
\hline IL-6 (pg/mL) & $11.65 \pm 1.17$ & $7.24 \pm 1.63$ & 0.017 * & $11.84 \pm 0.49$ & $11.82 \pm 1.16$ & 0.116 \\
\hline IL-10 (pg/mL) & $1.04 \pm 0.19$ & $9.91 \pm 2.04$ & $0.018 *$ & $1.56 \pm 0.13$ & $9.20 \pm 5.00$ & 0.153 \\
\hline $\mathrm{IL}-1 \beta(\mathrm{pg} / \mathrm{mL})$ & $7.79 \pm 0.76$ & $5.42 \pm 0.80$ & $0.008 *$ & $6.97 \pm 0.64$ & $6.29 \pm 0.39$ & 0.117 \\
\hline $\mathrm{TNF}-\alpha(\mathrm{pg} / \mathrm{mL})$ & $13.75 \pm 2.93$ & $7.59 \pm 1.54$ & 0.011 * & $9.25 \pm 0.90$ & $9.22 \pm 0.56$ & 0.679 \\
\hline $\operatorname{IgA}(\mathrm{ng} / \mathrm{mL})$ & $521.02 \pm 69.33$ & $636.48 \pm 79.23$ & $0.004 *$ & $579.40 \pm 54.02$ & $504.73 \pm 60.96$ & 0.877 \\
\hline $\mathrm{hsCRP}(\mathrm{ml} / \mathrm{L})$ & $0.017 \pm 0.006$ & $0.008 \pm 0.002$ & 0.086 & $0.012 \pm 0.001$ & $0.015 \pm 0.001$ & 0.078 \\
\hline LPS (pg/mL) & $108.99 \pm 9.62$ & $55.00 \pm 6.09$ & $<0.001 *$ & $93.92 \pm 7.87$ & $81.42 \pm 6.18$ & 0.054 \\
\hline ZO-1 (ng/mL) & $1.37 \pm 0.17$ & $0.98 \pm 0.18$ & $0.032 *$ & $1.42 \pm 0.17$ & $1.41 \pm 0.16$ & 0.551 \\
\hline
\end{tabular}

$*$ = Significant difference in $p$-value at 95\% confidence interval. HDL = High-Density Lipoprotein; LDL = Low-Density Lipoprotein; FBS = Fasting Blood Sugar; IL = Interleukin; TNF- $\alpha=$ Tumor Necrosis Factor alpha; IgA = Immunoglobulin A; hsCRP = High Sensitivity C-Reactive Protein; LPS = Lipopolysaccharide; $\mathrm{ZO}=$ zonulin.

Table 3. Changes in the studied parameters within groups at different times, expressed as mean \pm SE.

\begin{tabular}{|c|c|c|c|c|c|c|}
\hline \multirow{2}{*}{ Parameters } & \multicolumn{2}{|c|}{ Synbiotic $(\mathbf{N}=36)$} & \multirow{2}{*}{$p$-Value } & \multicolumn{2}{|c|}{ Placebo $(\mathrm{N}=36)$} & \multirow{2}{*}{$p$-Value } \\
\hline & Baseline & 12 Weeks & & Baseline & 12 Weeks & \\
\hline Lactulose & $0.16 \pm 0.03$ & $0.07 \pm 0.02$ & $<0.001 *$ & $0.12 \pm 0.03$ & $0.08 \pm 0.02$ & 0.135 \\
\hline Lactulose/mannitol ratio & $0.20 \pm 0.06$ & $0.09 \pm 0.01$ & 0.072 & $0.14 \pm 0.02$ & $0.12 \pm 0.02$ & 0.315 \\
\hline QA $(\mathrm{ng} / \mathrm{mL})$ & $23.53 \pm 2.42$ & $13.75 \pm 1.71$ & $<0.001 *$ & $22.44 \pm 1.69$ & $24.25 \pm 1.46$ & 0.375 \\
\hline 5-HIAA (mg/L) & $5.04 \pm 1.12$ & $9.61 \pm 1.95$ & 0.051 & $4.00 \pm 0.66$ & $4.95 \pm 0.93$ & 0.642 \\
\hline QA/5-HIAA Ratio & $3.14 \pm 1.60$ & $1.04 \pm 0.46$ & $0.008^{*}$ & $5.76 \pm 2.23$ & $4.71 \pm 1.77$ & 0.756 \\
\hline Cresol (umol/g sample) & $0.24 \pm 0.03$ & $0.09 \pm 0.05$ & $0.017 *$ & $0.31 \pm 0.16$ & $0.14 \pm 0.05$ & 0.225 \\
\hline Indole (umol/g sample) & $0.06 \pm 0.01$ & $0.04 \pm 0.00$ & $0.035 *$ & $0.11 \pm 0.06$ & $0.06 \pm 0.02$ & 0.18 \\
\hline Skatole (umol/g sample) & $0.07 \pm 0.03$ & $0.04 \pm 0.00$ & 0.285 & $0.05 \pm 0.03$ & $0.15 \pm 0.07$ & 0.285 \\
\hline Butyric acid (mmol/g sample) & $38.01 \pm 8.59$ & $93.80 \pm 18.96$ & $0.002 *$ & $46.40 \pm 12.29$ & $80.03 \pm 32.27$ & 0.311 \\
\hline Propionic acid (mmol/g sample) & $259.16 \pm 38.67$ & $624.12 \pm 82.82$ & $<0.001 *$ & $209.44 \pm 72.32$ & $466.52 \pm 178.52$ & 0.124 \\
\hline Acetic acid (mmol/g sample) & $202.63 \pm 37.70$ & $425.89 \pm 50.86$ & $<0.001 *$ & $206.56 \pm 61.60$ & $400.27 \pm 69.40$ & 0.161 \\
\hline Lactic acid (mmol/g sample) & $54.42 \pm 17.98$ & $175.81 \pm 36.88$ & $0.002 *$ & $92.11 \pm 53.12$ & $140.03 \pm 57.00$ & 0.866 \\
\hline TAC $(\mu \mathrm{mol} / \mathrm{mL})$ & $0.195 \pm 0.003$ & $0.200 \pm 0.011$ & 0.664 & $0.180 \pm 0.012$ & $0.193 \pm 0.008$ & 0.08 \\
\hline $\operatorname{MDA}(\mu \mathrm{mol} / \mathrm{mL})$ & $0.45 \pm 0.06$ & $0.53 \pm 0.09$ & 0.301 & $0.52 \pm 0.04$ & $0.45 \pm 0.03$ & 0.157 \\
\hline $\mathrm{GSH}(\mu \mathrm{g} / \mathrm{mL})$ & $44.99 \pm 17.46$ & $30.98 \pm 10.63$ & 0.075 & $24.87 \pm 6.86$ & $17.29 \pm 8.83$ & 0.08 \\
\hline Total SOD (Units/mL enzyme) & $56.91 \pm 5.52$ & $57.30 \pm 6.28$ & 0.854 & $54.01 \pm 11.78$ & $59.29 \pm 7.24$ & 0.492 \\
\hline Cu,Zn-SOD (Units / mL enzyme) & $16.93 \pm 3.13$ & $44.57 \pm 17.96$ & 0.345 & $35.28 \pm 7.34$ & $39.49 \pm 19.28$ & 0.686 \\
\hline
\end{tabular}

* = Significant difference in $p$-value at 95\% confidence interval. QA = Quinolinic acid; 5-HIAA = 5-Hydroxyindoleacetic acid; TAC = Total Antioxidant Capacity; MDA = Malondialdehyde; GSH = Glutathione Reduced; SOD = Superoxide Dismutase.

A Gaussian regression analysis of the data suggested that the synbiotic supplementation for 12 weeks significantly altered the body weight, body fat, muscle content, BMR, waist circumference, IL-6, IL-1 $\beta$, TNF- $\alpha$, LPS, ZO-1, lactulose/mannitol ratio, QA, 5-HIAA, $\mathrm{QA} / 5$-HIAA ratio, and butyric acid. There were no significant changes observed in cholesterol and antioxidant profiles (Table 6). 
Table 4. Comparison of the changes in studied parameters between groups. Changes represent the difference between baseline and at the end of the study.

\begin{tabular}{|c|c|c|c|}
\hline \multirow{2}{*}{ Parameters } & \multicolumn{2}{|c|}{ Baseline-12 Weeks } & \multirow{2}{*}{$p$-Value } \\
\hline & Synbiotic $(\mathrm{N}=36)$ & Placebo $(\mathrm{N}=36)$ & \\
\hline Body weight, cm & -1.64 & -0.46 & $0.002 *$ \\
\hline Body mass index, $\mathrm{kg} / \mathrm{m}^{2}$ & -0.39 & 0.13 & 0.128 \\
\hline Body fat, \% & -1.13 & 0.92 & 0.068 \\
\hline Visceral fat, $\%$ & -0.32 & 0.19 & 0.242 \\
\hline Muscle, $\%$ & -0.40 & -0.18 & 0.448 \\
\hline BMR (kcal) & 1.85 & -13.36 & 0.483 \\
\hline Arm circumference, $\mathrm{cm}$ & -0.12 & -0.11 & 0.809 \\
\hline Waist circumference, $\mathrm{cm}$ & -1.97 & -0.45 & 0.113 \\
\hline Hip circumference, $\mathrm{cm}$ & -0.59 & -0.49 & 0.51 \\
\hline Waist/hip ratio & -0.014 & 0.001 & 0.604 \\
\hline Total cholesterol (mg/dL) & -5.47 & -3.33 & 0.695 \\
\hline Triglyceride (mg/dL) & -4.28 & 1.24 & 0.521 \\
\hline HDL-cholesterol (mg/dL) & 2.9 & 0.48 & 0.066 \\
\hline LDL-cholesterol (mg/dL) & -11.28 & -6.87 & 0.599 \\
\hline FBS $(\mathrm{mg} / \mathrm{dL})$ & -2.79 & 8.5 & $0.043 *$ \\
\hline IL-6 (pg/mL) & -4.41 & -0.02 & 0.010 * \\
\hline IL-10 (pg/mL) & 8.87 & 7.64 & 0.142 \\
\hline $\mathrm{IL}-1 \beta(\mathrm{pg} / \mathrm{mL})$ & -2.37 & -0.69 & $0.041^{*}$ \\
\hline TNF- $\alpha(\mathrm{pg} / \mathrm{mL})$ & -6.16 & -0.04 & 0.005 * \\
\hline $\operatorname{IgA}(\mathrm{ng} / \mathrm{mL})$ & 115.46 & -74.67 & 0.049 * \\
\hline $\mathrm{hsCRP}(\mathrm{ml} / \mathrm{L})$ & -0.009 & 0.003 & $0.002 *$ \\
\hline LPS (pg/mL) & -53.99 & -12.50 & $0.002 *$ \\
\hline $\mathrm{ZO}-1$ (ng/mL) & -0.39 & -0.01 & 0.061 \\
\hline
\end{tabular}

$*$ = Significant difference in $p$-value at $95 \%$ confidence interval. HDL $=$ High-Density Lipoprotein LDL = Low-Density Lipoprotein; FBS = Fasting Blood Sugar; IL = Interleukin; TNF- $\alpha=$ Tumor Necrosis Factor alpha; IgA = Immunoglobulin A; hsCRP = High Sensitivity C-Reactive Protein; LPS = Lipopolysaccharide; $\mathrm{ZO}=$ zonulin.

Table 5. Comparison of the changes in studied parameters between groups. Changes represent the difference between baseline and at the end of the study.

\begin{tabular}{|c|c|c|c|}
\hline \multirow{2}{*}{ Parameters } & \multicolumn{2}{|c|}{ Baseline-12 Weeks } & \multirow{2}{*}{$p$-Value } \\
\hline & Synbiotic $(\mathrm{N}=36)$ & Placebo $(\mathrm{N}=36)$ & \\
\hline Lactulose & -0.08 & -0.04 & $0.002 *$ \\
\hline Lactulose/mannitol ratio & -0.11 & -0.02 & 0.508 \\
\hline QA (ng/mL) & -9.78 & 1.8 & $<0.001 *$ \\
\hline 5-HIAA (mg/L) & 4.58 & 0.94 & 0.157 \\
\hline QA/5-HIAA Ratio & -2.10 & -1.05 & 0.095 \\
\hline Cresol (umol/g sample) & -0.15 & -0.16 & 0.661 \\
\hline Indole (umol/g sample) & -0.03 & -0.05 & 0.379 \\
\hline Skatole (umol/g sample) & -0.031 & 0.103 & 0.121 \\
\hline Butyric acid (mmol/g sample) & 55.79 & 33.64 & 0.229 \\
\hline Propionic acid (mmol/g sample) & 364.96 & 257.09 & 0.258 \\
\hline Acetic acid (mmol/g sample) & 223.25 & 193.71 & 0.47 \\
\hline Lactic acid (mmol/g sample) & 121.39 & 47.92 & 0.162 \\
\hline $\mathrm{TAC}(\mu \mathrm{mol} / \mathrm{mL})$ & 0.005 & 0.013 & 0.557 \\
\hline $\operatorname{MDA}(\mu \mathrm{mol} / \mathrm{mL})$ & 0.08 & -0.07 & 0.117 \\
\hline $\mathrm{GSH}(\mu \mathrm{g} / \mathrm{mL})$ & -14.01 & -7.59 & 0.584 \\
\hline Total SOD (Units/mL enzyme) & 0.39 & 5.28 & 0.917 \\
\hline $\mathrm{Cu}, \mathrm{Zn}$-SOD (Units/mL enzyme) & 27.64 & 4.21 & 0.251 \\
\hline
\end{tabular}

* = Significant difference in $p$-value at 95\% confidence interval. QA = Quinolinic acid; 5-HIAA = 5Hydroxyindoleacetic acid; TAC = Total Antioxidant Capacity; MDA = Malondialdehyde; GSH = Glutathione Reduced; SOD = Superoxide Dismutase. 
Table 6. Gaussian regression analysis summary at week 12 of supplementation for synbiotic group.

\begin{tabular}{|c|c|c|c|}
\hline Parameter & Coefficient & $95 \% \mathrm{CI}$ & $p$-Value \\
\hline Body weight, cm & -1.76 & $(-3.17$ to -0.34$)$ & $0.018^{*}$ \\
\hline Body mass index, $\mathrm{kg} / \mathrm{m}^{2}$ & 0.123 & $(-0.64$ to 0.88$)$ & 0.744 \\
\hline Body fat, $\%$ & -2.55 & $(-4.74$ to -0.37$)$ & $0.023 *$ \\
\hline Visceral fat, $\%$ & -0.17 & $(-0.96$ to 0.61$)$ & 0.651 \\
\hline Muscle, \% & -5.13 & $(-8.82$ to -1.44$)$ & $0.027 *$ \\
\hline BMR (kcal) & 57.27 & $(2.77$ to 111.76$)$ & $0.040 *$ \\
\hline Arm circumference, $\mathrm{cm}$ & -0.14 & $(-2.61$ to 2.33$)$ & 0.909 \\
\hline Waist circumference, $\mathrm{cm}$ & -2.73 & $(-5.23$ to -0.23$)$ & 0.033 * \\
\hline Hip circumference, $\mathrm{cm}$ & -4.54 & $(-10.06$ to 0.97$)$ & 0.103 \\
\hline Waist/hip ratio & -0.02 & $(-0.05$ to 0.01$)$ & 0.131 \\
\hline Total cholesterol (mg/dL) & -8.01 & $(-26.91$ to 10.90$)$ & 0.397 \\
\hline Triglyceride (mg/dL) & -0.02 & $(-23.02$ to 22.97$)$ & 0.998 \\
\hline HDL-cholesterol (mg/dL) & 3.22 & $(-1.84$ to 8.27$)$ & 0.207 \\
\hline LDL-cholesterol (mg/dL) & -10.57 & $(-26.42$ to 5.28$)$ & 0.186 \\
\hline FBS (mg/dL) & -2.24 & $(-16.42$ to 11.94$)$ & 0.751 \\
\hline IL-6 (pg/mL) & -4.50 & $(-8.78$ to -0.23$)$ & 0.040 * \\
\hline IL-10 (pg/mL) & 5.18 & $(-9.96$ to 20.32$)$ & 0.477 \\
\hline $\mathrm{IL}-1 \beta(\mathrm{pg} / \mathrm{mL})$ & -1.43 & $(-2.78$ to -0.08$)$ & $0.039 *$ \\
\hline TNF- $\alpha(\mathrm{pg} / \mathrm{mL})$ & -4.26 & $(-6.51$ to -2.01$)$ & $0.001 *$ \\
\hline $\operatorname{IgA}(\mathrm{ng} / \mathrm{mL})$ & 117.99 & $(-55.97$ to 291.95$)$ & 0.179 \\
\hline $\operatorname{hsCRP}(\mathrm{ml} / \mathrm{L})$ & -0.003 & $(-0.011$ to 0.005$)$ & 0.497 \\
\hline LPS (pg/mL) & -32.59 & $(-53.68$ to -11.49$)$ & 0.004 * \\
\hline $\mathrm{ZO}-1(\mathrm{ng} / \mathrm{mL})$ & -0.57 & $(-1.08$ to -0.06$)$ & $0.032 *$ \\
\hline Lactulose & -0.02 & $(-0.07$ to 0.02$)$ & 0.319 \\
\hline Lactulose/mannitol ratio & -0.12 & $(-0.20$ to -0.04$)$ & 0.008 * \\
\hline $\mathrm{QA}(\mathrm{ng} / \mathrm{mL})$ & -8.22 & $(-16.04$ to -0.40$)$ & $0.041 *$ \\
\hline 5-HIAA (mg/L) & 8.59 & $(0.68$ to 16.50$)$ & 0.036 * \\
\hline QA/5-HIAA Ratio & -7.15 & $(-13.69$ to -0.61$)$ & $0.035 *$ \\
\hline Cresol (umol/g sample) & 0.09 & $(-0.30$ to 0.48$)$ & 0.583 \\
\hline Indole (umol/g sample) & -0.004 & $(-0.054$ to 0.046$)$ & 0.865 \\
\hline Skatole (umol/g sample) & -0.47 & $(-2.07$ to 1.12$)$ & 0.165 \\
\hline Butyric acid (mmol/g sample) & 59.74 & (20.30 to 99.17$)$ & $0.009 *$ \\
\hline Propionic acid (mmol/g sample) & -171.28 & $(-541.06$ to 198.51$)$ & 0.335 \\
\hline Acetic acid (mmol/g sample) & -111.03 & ( -324.73 to 102.68$)$ & 0.28 \\
\hline Lactic acid (mmol/g sample) & 6.73 & ( -135.27 to 148.73$)$ & 0.919 \\
\hline $\mathrm{TAC}(\mu \mathrm{mol} / \mathrm{mL})$ & -0.03 & $(-0.09$ to 0.04$)$ & 0.284 \\
\hline $\mathrm{MDA}(\mathrm{umol} / \mathrm{mL})$ & 0.43 & $(-0.36$ to 1.22$)$ & 0.18 \\
\hline $\mathrm{GSH}(\mu \mathrm{g} / \mathrm{mL})$ & 7.48 & ( -6.37 to 21.33$)$ & 0.208 \\
\hline Total SOD (Units /mL enzyme) & -32.33 & $(-91.18$ to 26.52$)$ & 0.179 \\
\hline $\mathrm{Cu}, \mathrm{Zn}$-SOD (Units / $\mathrm{mL}$ enzyme) & -74.39 & $(-203.46$ to 54.67$)$ & 0.131 \\
\hline
\end{tabular}

* = Significantly difference in $p$-value at $95 \%$ confidence interval. Compare with the placebo group at week 12, HDL = High-Density Lipoprotein; LDL = Low-Density Lipoprotein; FBS = Fasting Blood Sugar; $\mathrm{IL}=$ Interleukin; TNF- $\alpha=$ Tumor Necrosis Factor alpha; IgA = Immunoglobulin A; hsCRP = High Sensitivity C-Reactive Protein; LPS = Lipopolysaccharide; ZO = zonulin; QA = Quinolinic acid; 5-HIAA = 5Hydroxyindoleacetic acid; TAC = Total Antioxidant Capacity; MDA = Malondialdehyde; GSH = Glutathione Reduced; SOD = Superoxide Dismutase.

\section{Discussion}

The synergistic blend of both prebiotics and probiotics reduces plasma fasting insulin [25]. The most-used prebiotics are arabinoxylan and fructans [26]. The synbiotic supplementation of Bifidobacteria strains along with galactooligosaccharide may improve intestinal barrier function and possess anti-obesity effects [27].

There is a need for more approaches to aid in weight loss or to control obesity. Supplementation with Lactobacillus plantarum in obese mice reduced the deposition of adipose and upregulated the expression of lipid oxidative genes compared to control mice [28]. In order to treat obesity, Lactobacillus species can be used in combination with dietary management. L. sakei was found to impose anti-obesity effects when used in obese murine models [29,30]. The synbiotic supplements contained L. acidophilus, Bifidobacterium lactis, B. longum and B. bifidum as well as prebiotic galactooligosaccharide mixture, which increased the abundance of gut microbiome and also improved markers of metabolic syndrome as well as immune function in obese adults [31-34]. The supplementation of L. gasseri SBT2055mediated fermented milk for 12 weeks reduced the weight and the abdominal visceral and subcutaneous fat mass in obese human subjects [35]. 
Treating obesity has been a long-term-but not well-defined-methodology that has been linked with gut microbial management. Even though there have been numerous research works carried out on obesity, the clarification needed regarding the treatment of obesity remains lacking. The present study was performed to inspect the impact of the supplementation of a synbiotics preparation containing L. paracasei, B. longum, B. breve, inulin, and fructooligosaccharide on body composition and metabolic biomarkers in Thai obese subjects.

The supplementation of pro-, pre-, and synbiotics to an organism might alter the secretion of some hormones and neurotransmitters as well as inflammatory factors that inhibit the avidity towards food, therefore reducing weight gain [36]. Many systemic reviews and meta-analyses provide evidence about synbiotics intake assisting the lipid profile and improving dyslipidemia [37]. Synbiotic supplements and foods potentially modulate the gut microbiota as well as improving the metabolism of lipids, insulin resistance, and liver enzymes to a greater extent than either pro- or prebiotics alone [38].

A well-known characteristic of probiotics is their involvement in an improved serum lipid profile through immunomodulatory properties [39]. They also may reduce inflammatory cytokines and Toll-like receptor 4 (TLR-4) activation, leading to a great impact on the serum lipid profile [40]. Probiotics integrate cholesterol in their cellular membrane [41] and convert it into coprostanol [42], resulting in a reduction in cholesterol absorption and serum total cholesterol levels by means of higher bile salt excretion $[43,44]$. It is a well-known fact that probiotics supplementation can modulate body weight and BMI if the tested individuals are treated for a longer duration. In addition to this, previous study suggests that the outcomes in weight reduction could be effective when prebiotics and probiotics are used together [45].

Overall findings from animal and human studies revealed the more beneficial functions of synbiotics in weight reduction and the modulation of the gut microbiome $[27,46]$ compared to prebiotics and probiotics alone [47-49].

Obese individuals showed low-grade inflammation because of the increased production of cytokines, C-reactive proteins (CRP), interleukins (IL), tumor necrosis factor (TNF), and lipopolysaccharides (LPS) [50,51], which in turn resulted in metabolic dysfunction and obesity-linked disorders [52].

The dietary supplementation of synbiotics prepared using L. gasseri and galactomannan and inulin fibers reduced the weight and anti-inflammatory effects of synbiotic preparations along with L. rhamnosus (CGMCC 1.3724), L. plantarum, L. paracasei F19, L. acidophilus and LactisBb12, which together with oligo fructose and inulin showed beneficial effects on waist and hip circumference and BMI in obese people [53].

The randomized controlled trails in obese and prediabetes subjects showed variable results such as reduced TC, TG [31,54], and LDL levels [54,55], and the inflammation markers hs-CRP, TNF, LPS, and MDA were also found to be reduced [55-57]. Hotamisligil [58] and Lubberts [59] demonstrated that obese individuals express more TNF- $\alpha$ mRNA and protein when compared to lean controls. Thus, the increase in TNF- $\alpha$ induced IL-6 and IL-7 gene expression [60]. So far, the gathered evidence substantiates the role of peripheral 5-hydroxyindole-3-acetic acid (5-HIAA), the derivative end product of serotonin (5-HT) that is also involved in the pathogenesis of obesity and abnormal lipid and glucose metabolism [61]. In addition, 5-HIAA is associated with chronic low-grade inflammation, which in turn leads to metabolic syndrome. There is a strong association between serum 5-HIAA and central obesity [61]. However, 5-HT has long been known to be involved in the control of appetite, energy balance, and weight control $[62,63]$. Kinoshita and colleagues proved that 5-HT is responsible for adipocyte differentiation and might lead to adipogenesis and obesity [64]. Kim and colleagues showed that 5-HIAA is directly correlated with low-glyceride levels. Furthermore, there is a negative correlation between HDL cholesterol and 5-HIAA. In addition, an increase in 5-HIAA concentration increases plasma triglyceride levels, but the HDL cholesterol remains unaltered. Similarly, higher 5-HT concentrations were also detected in the blood of high-fat-diet-fed mice [65]. It is 
a well-known fact that zonulin is the physiological modulator of intestinal permeability and also a serum biomarker for impaired intestinal permeability [66-68]. The zonulin level was found to be elevated above the reference value in individuals with morbid obesity. Szonulin was partially controlled after a 6-month-long conservative weight loss intervention and further reduced after bariatric surgery [69].

A meta-analysis by Ramezani Ahmadi and colleagues suggested that, compared to placebo, supplementation with pro/synbiotics pointedly reduced the serum zonulin level among selected subjects. Due to the comparison between probiotics and synbiotics, the finding of a significant level of serum zonulin reduction was only in subjects treated with probiotics [70]. The role of IL-1 $\beta$ in regulating adipose inflammation and fat-liver cross talk has been questioned. IL- $1 \beta$ regulates the lipid storage capacity in adipose tissues of the liver; however, in its absence, the adipose tissue expands, increasing in response to excess calories [71]. However, it is clear that IL- $1 \beta$ is a major promoter of adipose tissue inflammation in obese subjects [72].

Our results shows that 12 weeks of synbiotics supplementation significantly reduced body weight, BMI and body fat, visceral fat, BMR, and arm, waist, and hip circumferences compared to the placebo group (Table 1) in Thai obese subjects. The same parameters showed significant reductions in different time periods as well (Table 2). Reductions in IL-6, IL-1 $\beta$, TNF- $\alpha$, LPS, ZO-1, lactulose/mannitol ratio, QA, 5-HIAA, QA/5-HIAA ratio, and butyric acid levels were observed in the 12-week synbiotics-supplemented group (Table 6). The results support the notion that the potential use of synbiotics could be a promising choice for the treatment and/or management of obesity. This study may stimulate interest in molecular underpinnings beyond these significant results. Moreover, the study shows that synbiotic involvements in treating obesity could be a hopeful suggestive therapy in obesity and other related metabolic disorders.

\section{Conclusions}

The intake of synbiotics for a stipulated period of time had a moderating effect on body weight, BMI, body fat, visceral fat, BMR, and arm, waist, and hip circumference. The effects of synbiotic supplementation were proven to greatly reduce the above-mentioned parameters when administered for prolonged period of time. This evidence suggests that synbiotic supplementation produces a stronger effect compared to separate prebiotic and probiotic treatments. Additional anti-obesity effects can be obtained when obese subjects carry out synbiotic supplementation alongside any physical activity. The present study demonstrated that 12 weeks of synbiotic supplementation significantly reduced the physical parameters as well as the inflammation markers IL-6, IL-1 $\beta$, TNF- $\alpha$ and other obesity markers including LPS, zonulin, 5-HIAA, and QA in Thai obese subjects. These obtained results offer a new platform to document other new markers and the effect of various other synbiotic supplementation combinations in the study of obesity.

Author Contributions: Conceptualization, C.C., B.S.S., S.S., and P.S.; methodology, N.K., S.K., S.S. and P.S.; formal analysis, N.K., S.K., S.P., and S.S.; investigation, C.C., B.S.S., S.S., and P.S.; writingoriginal draft preparation, B.S.S.; S.T.; writing—review and editing, C.C., B.S.S., P.K., S.T., A.B., K.C., S.S., and P.S.; project administration, C.C. and P.S. All authors have read and agreed to the published version of the manuscript.

Funding: This research was funded by the Research and Innovation Fund for Small Scale Enterprise (RISE) (Grant no. RDG62I0007). S.K. was funded by the Research and Researcher Development for Industry Program (RRi) of the Thailand Research Fund (TRF) (Grant no. PHD60I0022. The research was partially supported by Chiang Mai University.

Institutional Review Board Statement: The study was conducted following the Good Clinical Practices, fully complied with the ethical guidelines of a clinical trial, and conducted according to the Declaration of Helsinki; the Ethics Committee approved the protocol of Mae Fah Luang University (Code: REH-62151).

Informed Consent Statement: Informed consent was obtained from all subjects involved in the study. 
Data Availability Statement: The data presented in the manuscript is available on request from the corresponding author.

Acknowledgments: The authors gratefully acknowledge Chiang Mai University and Mae Fah Luang University for research support. We would like to gratefully acknowledge Lactomason Co., Ltd., Gyeongsangnam-do, South Korea for the probiotic supplements.

Conflicts of Interest: The authors declare no conflict of interest.

\section{References}

1. Sivamaruthi, B.S.; Kesika, P.; Suganthy, N.; Chaiyasut, C. A review on role of microbiome in obesity and antiobesity properties of probiotic supplements. Biomed. Res. Int. 2019, 2019, 3291367. [CrossRef] [PubMed]

2. Obesity and Overweight. Available online: https://www.who.int/en/news-room/fact-sheets/detail/obesity-and-overweight (accessed on 10 January 2021).

3. Ley, R.E.; Turnbaugh, P.J.; Klein, S.; Gordon, J.I. Human gut microbes associated with obesity. Nature 2006, 444, 1022-1023. [CrossRef] [PubMed]

4. Tremaroli, V.; Bäckhed, F. Functional interactions between the gut microbiota and host metabolism. Nature 2012, 489, 242-249. [CrossRef] [PubMed]

5. Nagpal, R.; Newman, T.M.; Wang, S.; Jain, S.; Lovato, J.F.; Yadav, H. Obesity-linked gut microbiome dysbiosis associated with derangements in gut permeability and intestinal cellular homeostasis independent of diet. J. Diabetes Res. 2018, $2018,3462092$. [CrossRef] [PubMed]

6. Boulangé, C.L.; Neves, A.L.; Chilloux, J.; Nicholson, J.K.; Dumas, M.E. Impact of the gut microbiota on inflammation, obesity, and metabolic disease. Genome Med. 2016, 8, 1-42. [CrossRef] [PubMed]

7. Sivamaruthi, B.S.; Prasanth, M.I.; Kesika, P.; Chaiyasut, C. Probiotics in human mental health and diseases-A mini review. Trop. J. Pharm. Res. 2019, 18, 889-895. [CrossRef]

8. Sivamaruthi, B.S.; Suganthy, N.; Kesika, P.; Chaiyasut, C. The role of microbiome, dietary supplements, and probiotics in autism spectrum disorder. Int. J. Environ. Res. Public Health 2020, 17, 2647. [CrossRef]

9. Sivamaruthi, B.S.; Kesika, P.; Chaiyasut, C. The role of probiotics in colorectal cancer management. Evid. Based Complement. Alternat. Med. 2020, 2020, 3535982. [CrossRef]

10. De Vrese, M.; Schrezenmeir, A.J. Probiotics, prebiotics, and synbiotics. In Food Biotechnology; Springer: Berlin/Heidelberg, Germany, 2008; pp. 1-66.

11. Saghaei, M. Random allocation software for parallel group randomized trials. BMC Med. Res. Methodol. 2004, 4, 1-6. [CrossRef]

12. Minami, J.; Iwabuchi, N.; Tanaka, M.; Yamauchi, K.; Xiao, J.Z.; Abe, F.; Sakane, N. Effects of Bifidobacterium breve B-3 on body fat reductions in pre-obese adults: A randomized, double-blind, placebo-controlled trial. Biosci. Microbiota Food Health. 2018, 37, 67-75. [CrossRef]

13. Sang Gil, L.; Taoran, W.; Terrence, M.V.; Patrice, H.; Dae-Ok, K.; Sung, I.K. Validation of analytical methods for plasma total antioxidant capacity by comparing with urinary 8-isoprostane level. J. Microbiol. Biotechnol. 2017, 27, 388-394.

14. Kambayashi, Y.; Binh, N.T.; Asakura, H.W.; Hibino, Y.; Hitomi, Y.; Nakamura, H.; Ogino, K. Efficient assay for total antioxidant capacity in human plasma using a 96-well microplate. J. Clin. Biochem. Nutr. 2009, 44, 46-51. [CrossRef]

15. Zeb, A.; Ullah, F. A Simple Spectrophotometric Method for the Determination of Thiobarbituric Acid Reactive Substances in Fried Fast Foods. J. Anal. Methods Chem. 2016, 2016, 9412767. [CrossRef] [PubMed]

16. Atasayar, S.; Orhan, H. Malondialdehyde quantification in blood plasma of tobacco smokers and non-smokers. Fabad J. Pharm. Sci. 2004, 29, 15-19.

17. Joe, M.M.; Irwin, F. Superoxide Dismutase. J. Biol. Chem. 1969, 224, 6049-6065.

18. Rahman, I.; Kode, A.; Biswas, S.K. Assay for quantitative determination of glutathione and glutathione disulfide levels using enzymatic recycling method. Nat. Protoc. 2006, 1, 3159-3165. [CrossRef] [PubMed]

19. Kotani, A.; Miyaguchi, Y.; Kohama, M.; Ohtsuka, T.; Shiratori, T.; Kusu, F. Determination of short-chain fatty acids in rat and human feces by high-performance liquid chromatography with electrochemical detection. Anal. Sci. 2009, 25, $1007-1011$. [CrossRef] [PubMed]

20. Torii, T.; Kanemitsu, K.; Wada, T.; Itoh, S.; Kinugawa, K.; Hagiwara, A. Measurement of short-chain fatty acids in human faeces using high-performance liquid chromatography: Specimen stability. Ann. Clin. Biochem. 2010, 47, 447-452. [CrossRef]

21. Birkett, A.M.; Jones, G.P.; Muir, J.G. Simple high-performance liquid chromatographic analysis of phenol and p-cresol in urine and feces. J. Chromatogr. B Biomed. Sci. Appl. 1995, 674, 187-191. [CrossRef]

22. Chen, G.; Zamaratskaia, G.; Andersson, H.K.; Lundström, K. Effects of raw potato starch and live weight on fat and plasma skatole, indole and androstenone levels measured by different methods in entire male pigs. Food Chem. 2007, 101, 439-448. [CrossRef]

23. Nowak, A.; Libudzisz, Z. Ability of intestinal lactic bacteria to bind or/and metabolise phenol and p-cresol. Ann. Microbiol. 2007, 57, 329-335. [CrossRef]

24. Sequeira, I.R.; Lentle, R.G.; Kruger, M.C.; Hurst, R.D. Standardising the lactulose mannitol test of gut permeability to minimise error and promote comparability. PLoS ONE 2014, 9, e99256. [CrossRef] [PubMed] 
25. Barengolts, E. Gut microbiota, prebiotics, probiotics, and synbiotics in management of obesity and prediabetes: Review of randomized controlled trials. Endocr. Pract. 2016, 22, 1224-1234. [CrossRef] [PubMed]

26. Raninen, K.; Lappi, J.; Mykkänen, H.; Poutanen, K. Dietary fiber type reflects physiological functionality: Comparison of grain fiber, inulin, and polydextrose. Nutr. Rev. 2011, 69, 9-21. [CrossRef] [PubMed]

27. Vallianou, N.; Stratigou, T.; Christodoulatos, G.S.; Tsigalou, C.; Dalamaga, M. Probiotics, Prebiotics, Synbiotics, Postbiotics, and Obesity: Current evidence, controversies, and perspectives. Curr. Obes. Rep. 2020, 9, 179-192. [CrossRef] [PubMed]

28. Park, S.; Bae, J.H. Probiotics for weight loss: A systematic review and meta-analysis. Nutr. Res. 2015, 35, 566-575. [CrossRef] [PubMed]

29. Ji, Y.; Chung, Y.M.; Park, S.; Jeong, D.; Kim, B.; Holzapfel, W.H. Dose-dependent and strain-dependent anti-obesity effects of Lactobacillus sakei in a diet induced obese murine model. PeerJ 2019, 7, e6651. [CrossRef]

30. Ji, Y.; Park, S.; Chung, Y.; Kim, B.; Park, H.; Huang, E.; Jeong, D.; Jung, H.; Kim, B.; Hyun, C.; et al. Amelioration of obesity-related biomarkers by Lactobacillus sakei CJLS03 in a high-fat diet-induced obese murine model. Sci. Rep. 2019, 9, 6821. [CrossRef]

31. Vulevic, J.; Juric, A.; Tzortzis, G.; Gibson, G.R. A mixture of trans-galactooligosaccharide reduces markers of metabolic syndrome and modulates the fecal microbiota and immune function of overweight adults. J. Nutr. 2013, 143, 324-331. [CrossRef]

32. Canfora, E.E.; van der Beek, C.M.; Hermes, G.; Goossens, G.H.; Jocken, J.; Holst, J.J.; van Eijk, H.M.; Venema, K.; Smidt, H.; Zoetendal, E.G.; et al. Supplementation of diet with galacto-oligosaccharides increases bifidobacteria, but not insulin sensitivity, in obese prediabetic individuals. Gastroenterology 2017, 153, 87-97. [CrossRef]

33. Azcarate-Peril, M.A.; Butz, N.; Cadenas, M.B.; Koci, M.; Ballou, A.; Mendoza, M.; Ali, R.; Hassan, H. An attenuated Salmonella enterica Serovar Typhimurium strain and galacto-oligosaccharides accelerate clearance of salmonella infections in poultry through modifications to the gut microbiome. Appl. Environ. Microbiol. 2018, 84, e02526-17. [CrossRef] [PubMed]

34. Sergeev, I.N.; Aljutaily, T.; Walton, G.; Huarte, E. Effects of Synbiotic Supplement on Human Gut Microbiota, Body Composition and Weight Loss in Obesity. Nutrients 2020, 12, 222. [CrossRef] [PubMed]

35. Kadooka, Y.; Sato, M.; Imaizumi, K.; Ogawa, A.; Ikuyama, K.; Akai, Y.; Okano, M.; Kagoshima, M.; Tsuchida, T. Regulation of abdominal adiposity by probiotics (Lactobacillus gasseri SBT2055) in adults with obese tendencies in a randomized controlled trial. Eur. J. Clin. Nutr. 2010, 64, 636-643. [CrossRef] [PubMed]

36. Aoun, A.; Darwish, F.; Hamod, N. The influence of the gut microbiome on obesity in adults and the role of probiotics, prebiotics, and synbiotics for weight loss. Prev. Nutr. Food Sci. 2020, 25, 113-123. [CrossRef] [PubMed]

37. Hadi, A.; Ghaedi, E.; Khalesi, S.; Pourmasoumi, M.; Arab, A. Effects of synbiotic consumption on lipid profile: A systematic review and meta-analysis of randomized controlled clinical trials. Eur. J. Nutr. 2020, 59, 2857-2874. [CrossRef]

38. Hadi, A.; Mohammadi, H.; Miraghajani, M.; Ghaedi, E. Efficacy of synbiotic supplementation in patients with nonalcoholic fatty liver disease: A systematic review and meta-analysis of clinical trials: Synbiotic supplementation and NAFLD. Crit. Rev. Food Sci. Nutr. 2019, 59, 2494-2505. [CrossRef]

39. Konstantinov, S.R.; Smidt, H.; de Vos, W.M.; Bruijns, S.C.; Singh, S.K.; Valence, F.; Molle, D.; Lortal, S.; Altermann, E.; Klaenhammer, T.R.; et al. S layer protein A of Lactobacillus acidophilus NCFM regulates immature dendritic cell and T cell functions. Proc. Natl. Acad. Sci. USA 2008, 105, 19474-19479. [CrossRef]

40. Ouwehand, A.C.; Tiihonen, K.; Saarinen, M.; Putaala, H.; Rautonen, N. Influence of a combination of Lactobacillus acidophilus NCFM and lactitol on healthy elderly: Intestinal and immune parameters. Br. J. Nutr. 2008, 101, 367-375. [CrossRef]

41. Kimoto, H.; Ohmomo, S.; Okamoto, T. Cholesterol removal from media by Lactococci. J. Dairy Sci. 2002, 85, 3182-3188. [CrossRef]

42. Lye, H.S.; Rusul, G.; Liong, M.T. Removal of cholesterol by lactobacilli via incorporation and conversion to coprostanol. J. Dairy Sci. 2010, 93, 1383-1392. [CrossRef]

43. Begley, M.; Hill, C.; Gahan, C.G. Bile salt hydrolase activity in probiotics. Appl. Environ. Microbiol. 2006, 72, 1729-1738. [CrossRef] [PubMed]

44. Patel, A.K.; Singhania, R.R.; Pandey, A.; Chincholkar, S.B. Probiotic bile salt hydrolase: Current developments and perspectives. Appl. Biochem. Biotechnol. 2010, 162, 166-180. [CrossRef]

45. Wiciński, M.; Gębalski, J.; Gołębiewski, J.; Malinowski, B. Probiotics for the treatment of overweight and obesity in humans-A review of clinical trials. Microorganisms 2020, 8, 1148. [CrossRef] [PubMed]

46. Hofmann, D.E.; Fraser, C.M.; Palumbo, F.; Ravel, J.; Rowthorn, V.; Schwartz, J. Probiotics: Achieving a better regulatory ft. Food Drug Law J. 2014, 69, 237-272.

47. Crovesy, L.; Ostrowski, M.; Ferreira, D.; Rosado, E.L.; Soares-Mota, M. Effect of Lactobacillus on body weight and body fat in overweight subjects: A systematic review of randomized controlled clinical trials. Int. J. Obes. 2017, 41, 1607-1614. [CrossRef]

48. Krumbeck, J.A.; Rasmussen, H.E.; Hutkins, R.W.; Clarke, J.; Shawron, K.; Keshavarzian, A.; Walter, J. Probiotic Bifidobacterium strains and galacto-oligosaccharides improve intestinal barrier function in obese adults but show no synergism when used together as synbiotics. Microbiome 2018, 6, 121. [CrossRef]

49. Barathikannan, K.; Chelliah, R.; Rubab, M.; Daliri, E.B.; Elahi, F.; Kim, D.H.; Agastian, P.; Oh, S.Y.; Oh, D.H. Gut microbiome modulation based on probiotic application for anti-obesity: A review on efficacy and validation. Microorganisms 2019, 7, 456. [CrossRef]

50. Hotamisligil, G.S. Endoplasmic reticulum stress and inflammation in obesity and type 2 diabetes. Novartis Found. Symp. 2007, 286, 86-203. [PubMed] 
51. Hotamisligil, G.S.; Erbay, E. Nutrient sensing and inflammation in metabolic diseases. Nat. Rev. Immunol. 2008, 8, 923-934. [CrossRef]

52. Scarpellini, E.; Tack, J. Obesity and metabolic syndrome: An inflammatory condition. Dig. Dis. 2012, 30, 148-153. [CrossRef]

53. Ferrarese, R.; Ceresola, E.R.; Preti, A.; Canducci, F. Probiotics, prebiotics and synbiotics for weight loss and metabolic syndrome in the microbiome era. Eur. Rev. Med. Pharmacol. Sci. 2018, 22, 7588-7605.

54. Dehghan, P.; Pourghassem Gargari, B.; Asgharijafarabadi, M. Effects of high performance inulin supplementation on glycemic status and lipid profile in women with type 2 diabetes: A randomized, placebo-controlled clinical trial. Health Promot. Perspect. 2013, 3, 55-63.

55. Genta, S.; Cabrera, W.; Habib, N.; Pons, J.; Carillo, I.M.; Grau, A.; Sánchez, S. Yacon syrup: Beneficial effects on obesity and insulin resistance in humans. Clin. Nutr. 2009, 28, 182-187. [CrossRef]

56. Pourghassem Gargari, B.; Dehghan, P.; Aliasgharzadeh, A.; Asghari Jafar-Abadi, M. Effects of high performance inulin supplementation on glycemic control and antioxidant status in women with type 2 diabetes. Diabetes Metab. J. 2013, 37, 140-148. [CrossRef]

57. Dehghan, P.; Pourghassem Gargari, B.; Asghari Jafarabadi, M. Oligofructose-enriched inulin improves some inflammatory markers and metabolic endotoxemia in women with type 2 diabetes mellitus: A randomized controlled clinical trial. Nutrition 2014, 30, 418-423. [CrossRef]

58. Hotamisligil, G.S.; Arner, P.; Caro, J.F.; Atkinson, R.L.; Spiegelman, B.M. Increased adipose tissue expression of tumor necrosis factor-alpha in human obesity and insulin Resistance. J. Clin. Investig. 1995, 95, 2409-2415. [CrossRef]

59. Lubberts, E. I/-/H targeting: On the road to prevent chronic destructive arthritis? Cytokine 2008, 41, 84-91. [CrossRef]

60. Pang, G.; Couch, L.; Batey, R.; Clancy, R.; Cripps, A. GM-CSF, IL-1 alpha, IL-1 beta, IL-6, IL-8, IL-10, ICAM-1 and VCAM-1 gene expression and cytokine production in human duodenal fibroblasts stimulated with lipopolysaccharide, IL-1 alpha and TNFalpha. Clin. Exp. Immunol. 1994, 96, 437-443. [CrossRef]

61. Afarideh, M.; Behdadnia, A.; Noshad, S.; Mirmiranpour, H.; Mousavizadeh, M.; Khajeh, E.; Rad, M.V.; Mazaheri, T.; Nakhjavani, M.; Esteghamati, A. Association of Peripheral 5-Hydroxyindole-3-Acetic Acid, A serotonin derivative, with metabolic syndrome and low-grade inflammation. Endocr Pract. 2015, 21, 711-718. [CrossRef]

62. Tecott, L.H.; Sun, L.M.; Akana, S.F.; Strack, A.M.; Lowenstein, D.H.; Dallman, M.F.; Julius, D. Eating disorder and epilepsy in mice lacking 5-HT2c serotonin receptors. Nature 1995, 374, 542-546. [CrossRef]

63. Jonnakuty, C.; Gragnoli, C. What do we know about serotonin? J. Cell Physiol. 2008, 217, 301-306. [CrossRef]

64. Kinoshita, M.; Ono, K.; Horie, T.; Nagao, K.; Nishi, H.; Kuwabara, Y.; Takanabe-Mori, R.; Hasegawa, K.; Kita, T.; Kimura, T. Regulation of adipocyte differentiation by activation of serotonin (5-HT) receptors 5-HT2AR and 5-HT2CR and involvement of microRNA-448-mediated repression of KLF5. Mol. Endocrinol. 2010, 24, 1978-1987. [CrossRef]

65. Kim, H.J.; Kim, J.H.; Noh, S.; Hur, H.J.; Sung, M.J.; Hwang, J.T.; Park, J.H.; Yang, H.J.; Kim, M.S.; Kwon, D.Y.; et al. Metabolomic analysis of livers and serum from high-fat diet induced obese mice. J. Proteome Res. 2011, 10, 722-731. [CrossRef] [PubMed]

66. Wang, W.; Uzzau, S.; Goldblum, S.E.; Fasano, A. Human zonulin, a potential modulator of intestinal tight junctions. J. Cell Sci. 2000, 113 Pt 24, 4435-4440. [CrossRef]

67. Sapone, A.; de Magistris, L.; Pietzak, M.; Clemente, M.G.; Tripathi, A.; Cucca, F.; Lampis, R.; Kryszak, D.; Cartenì, M.; Generoso, M.; et al. Zonulin upregulation is associated with increased gut permeability in subjects with type 1 diabetes and their relatives. Diabetes 2006, 55, 1443-1449. [CrossRef] [PubMed]

68. Tripathi, A.; Lammers, K.M.; Goldblum, S.; Shea-Donohue, T.; Netzel-Arnett, S.; Buzza, M.S.; Antalis, T.M.; Vogel, S.N.; Zhao, A.; Yang, S.; et al. Identification of human zonulin, a physiological modulator of tight junctions, as prehaptoglobin-2. Proc. Natl. Acad. Sci. USA 2009, 106, 16799-16804. [CrossRef]

69. Aasbrenn, M.; Lydersen, S.; Farup, P.G. Changes in serum zonulin in individuals with morbid obesity after weight-loss interventions: A prospective cohort study. BMC Endocr Disord. 2020, 20, 108. [CrossRef]

70. Ramezani Ahmadi, A.; Sadeghian, M.; Alipour, M.; Ahmadi Taheri, S.; Rahmani, S.; Abbasnezhad, A. The effects of probiotic/synbiotic on serum level of zonulin as a biomarker of intestinal permeability: A systematic review and meta-analysis. Iran. J. Public Health 2020, 49, 1222-1231. [CrossRef]

71. Kamari, Y.; Shaish, A.; Vax, E.; Shemesh, S.; Kandel-Kfir, M.; Arbel, Y.; Olteanu, S.; Barshack, I.; Dotan, S.; Voronov, E.; et al. Lack of interleukin- $1 \alpha$ or interleukin- $1 \beta$ inhibits transformation of steatosis to steatohepatitis and liver fibrosis in hypercholesterolemic mice. J. Hepatol. 2011, 55, 1086-1094. [CrossRef]

72. Nov, O.; Shapiro, H.; Ovadia, H.; Tarnovscki, T.; Dvir, I.; Shemesh, E.; Kovsan, J.; Shelef, I.; Carmi, Y.; Voronov, E.; et al. Interleukin$1 \beta$ regulates fat-liver crosstalk in obesity by auto-paracrine modulation of adipose tissue inflammation and expandability. PLOS ONE 2013, 8, e53626. 